\title{
Freqüência de anticorpos irregulares em politransfundidos no Hemocentro Regional de Uberaba-MG, de 1997 a 2005
}

\author{
Frequency of irregular antibodies in multiple-transfused patients at the Regional Blood Bank of \\ Uberaba, from 1997 to 2005
}

Paulo Roberto J. Martins ${ }^{1}$

Vitor M. Alves ${ }^{2}$

Gilberto A. Pereira ${ }^{3}$

Helio Moraes-Souza ${ }^{4}$

\begin{abstract}
A fenotipagem eritrocitária pré-transfusional é um importante procedimento para aumentar a segurança das transfusões sangüíneas, sendo realizada rotineiramente no Hemocentro Regional de Uberaba-MG (HRU) desde 1996. O presente trabalho tem como objetivo geral avaliar a freqüência de anticorpos antieritrocitários irregulares em politransfundidos, de 1997 a 2005. Através de estudo retrospectivo foram levantados dados no arquivo do HRU de todos os pacientes aloimunizados, realizou-se análise estatística descritiva e comparam-se as proporções pelo teste " $Z$ ". Dos 23.220 transfundidos no perído, com média de 5,7 transfusões por paciente, observou-se a ocorrência de aloimunização em 173 (0,75\%). Os sistemas Rh e Kell juntos tiveram freqüencia superior a $70 \%$. A proporção do anti-D foi significativamente maior nas mulheres $(p<0,05)$ e não houve diferença no sistema Rh entre brancos e não-brancos. Quanto à faixa etária, $70 \%$ tinham mais de 30 anos. Dos 73 pacientes que tiveram a doença de base registrada, 39,73\% eram portadores de anemias agudas, 31,51\% de anemias crônicas e 28,77\% de doenças oncológicas ou onco-hematológicas. Aproximadamente $70 \%$ dos anticorpos foram identificados até a décima transfusão. A baixa ocorrência da aloimunização no HRU reforça a importância da fenotipagem eritrocitária para todos os pacientes dependentes de transfusões crônicas, bem como da sua implantação na rotina de todos os serviços de hemoterapia. Rev. bras. hematol. hemoter. 2008;30(4):272-276.
\end{abstract}

Palavras-chave: Aloimunização; anticorpos irregulares; pacientes politransfundidos.

\section{Introdução}

A descoberta dos antígenos eritrocitários foi considerada um dos avanços mais importantes nas pesquisas da área médica, na primeira metade do século XX. ${ }^{1}$ Desde que o sistema ABO foi descoberto, em 1900, foram descritos mais de 250 antígenos eritrocitários, organizados em sistemas, séries e coleções. ${ }^{16}$ Os anticorpos antieritrocitários classifi- cam-se em regulares e irregulares. Os primeiros se desenvolvem naturalmente após o nascimento, como anti-A, anti-B e anti-AB, todos do sistema $\mathrm{ABO}$. Os irregulares (sistemas Rh, Kell, MNS, Lewis, Duffy, Kidd e outros) se desenvolvem em decorrência de transfusões ou gestações incompatíveis, não sendo encontrados normalmente. ${ }^{2}$

$\mathrm{O}$ antígeno $\mathrm{D}$, do sistema $\mathrm{Rh}$, é considerado o mais imunogênico. Por esse motivo, a transfusão de sangue com-

\footnotetext{
${ }^{I}$ Professor Adjunto da Disciplina de Hematologia e Hemoterapia da Universidade Federal do Triângulo Mineiro (UFTM) e coordenador do Hemocentro Regional de Uberaba/Fundação Hemominas - Uberaba-MG.

${ }^{2}$ Biomédico graduado pela UFTM e bolsista de Iniciação Científica da Fapemig, através da Fundação Hemominas.

${ }^{3}$ Professor da disciplina de Bioestatística da UFTM - Uberaba-MG.

${ }^{4}$ Professor Titular da Disciplina de Hematologia e Hemoterapia da UFTM e pesquisador do Hemocentro Regional de Uberaba/Fundação

Hemominas - Uberaba-MG.

Universidade Federal do Triângulo Mineiro / Hemocentro Regional de Uberaba/Fundação Hemominas - Uberaba-MG.
}

Correspondência: Paulo Roberto Juliano Martins

Av. Getúlio Guaritá, $250-4^{\circ}$ andar - Bairro Abadia

38025-440 - Uberaba-MG - Brasil

Tel.: 553433125077

E-mail: hemocentro@mednet.com.br 
patível para esse antígeno tem se tornado uma prática habitual há várias décadas. Este fato, somado ao uso sistemático de gamaglobulina anti-D após as gestações de feto $\mathrm{RhD}$ incompatível, tem levado à redução dos casos de alossensibilização anti-D e da doença hemolítica do recém-nascido (DHRN). ${ }^{3}$ Contudo, com o aumento do emprego das transfusões sangüíneas em cirurgias, transplantes e tratamento clínico do câncer, a partir da década de oitenta, passou-se a observar um aumento da ocorrência de aloimunização nos indivíduos politransfundidos.

Schonewille et al., ${ }^{11}$ em um estudo restrito apenas a pacientes com doenças mielo e linfoproliferativas, encontraram um índice de 9,0\% de aloimunizados, no período de 1987 a 1996. Os enfermos receberam hemácias compatíveis apenas com os fenótipos ABO e D. García Gala et al., estudando pacientes com patologias hematológicas e nefrológicas, encontraram, entre 1990 e 1994, um índice de 1,28\% de aloimunizados. O sangue transfundido era compatível para os sistemas $\mathrm{ABO}$ e Rh.

A fenotipagem eritrocitária, introduzida gradativamente na rotina dos bancos de sangue, tem contribuído para reduzir o índice de aloimunização dos pacientes politransfundidos e aumentar a segurança transfusional. A introdução, pelo Hemocentro Regional de Uberaba (HRU), em 1996, da fenotipagem rotineira de pacientes com potencial de virem a receber múltiplas transfusões, motivou o desenvolvimento do presente estudo, com o objetivo de determinar a prevalência desta iatrogenia no Serviço.

O trabalho tem também como objetivos avaliar a freqüência de diferentes anticorpos antieritrocitários irregulares em politransfundidos do Hemocentro Regional de Uberaba-MG (HRU)/Fundação Hemominas, no período de 1997 a 2005, bem como a distribuição destes anticorpos quanto ao gênero, cor, idade, tipo de patologia de base e número de transfusões recebidas.

\section{Casuística e Método}

Foram levantadas fichas no arquivo do HRU dos pacientes nos quais foram encontrados anticorpos irregulares, entre todos os indivíduos transfundidos no período de 1997 a 2005. Em um banco de dados foram registradas informações como sexo, idade, cor, doença que levou à indicação das transfusões, número de transfusões recebidas e tipo de anticorpo identificado.

Os dados foram analisados segundo análise estatística descritiva, através de freqüências absolutas e percentuais e os resultados foram organizados em tabelas. Compararam-se as proporções pelo teste " $\mathrm{Z} "{ }^{18}$ Valores de $p$ menores que 0,05 foram considerados estatisticamente significantes.

\section{Resultados}

No período de 1997 a 2005 foram realizadas 133.561 trans- fusões em 23.220 indivíduos atendidos em vários hospitais de Uberaba-MG e/ou no pronto atendimento do HRU, média de 5,7 transfusões por paciente. Foram identificados anticorpos irregulares em 173 pacientes $(0,75 \%)$, correspondendo a uma aloimunização para cada 772 transfusões. Os anticorpos de maior freqüência foram: anti-D $(24,28 \%)$, anti-E $(18,50 \%)$, anti-K $(13,87 \%)$ e anti-M $(10,41 \%)$. Associações de diferentes anticorpos ocorreram em 36 indivíduos (20,81\%), sendo que a mais freqüente envolveu anti-D + anti-C, em $14(8,09 \%$ do total de aloimunizados) (Tabela 1).

Tabela 1. Principais anticorpos irregulares identificados em 173 pacientes no HRU, de 1997 a 2005

\begin{tabular}{lcc}
\hline Tipo de anticorpo & $\mathrm{n}^{\circ}$ & $\%$ \\
\hline Sistema Rh & 42 & 24,28 \\
$\quad$ anti-D & 32 & 18,50 \\
anti-E & 03 & 1,73 \\
anti-C & 02 & 1,16 \\
anti-C & & \\
Outros sistemas & 24 & 13,87 \\
anti-K (Kell) & 18 & 10,41 \\
anti-M (MNS) & 05 & 2,89 \\
anti-Le ${ }^{\text {a }(\text { Lewis) }}$ & 05 & 2,89 \\
anti-Fy (Duffy) & 06 & 3,47 \\
outros anticorpos* & & \\
Associações & 14 & 8,09 \\
anti-D + anti-C & 22 & 12,72 \\
outras associações** & 173 & 100,00 \\
\hline Total &
\end{tabular}

*outros anticorpos: anti-Js (sistema Kell), anti-N e anti-S (sistema MNS), anti-Le ${ }^{b}$ (sistema Lewis), anti-Jk ${ }^{\mathrm{a}}$ e anti-Jk ${ }^{\mathrm{b}}$ (sistema Kidd).

${ }^{* *}$ outras associações: anti-C + anti-E, anti-C + anti-e, anti-E + antiC, anti-E + anti-K, anti-E + anti-Le ${ }^{\mathrm{a}}$, anti-E + anti-M, anti-E + anti-Fy ${ }^{\mathrm{a}}$, anti-E + anti-Jka, anti-E + anti-s, anti-c + anti-Fy ${ }^{a}$, anti-c + anti-Jka, anti-K + anti-Le ${ }^{\text {a }}$, anti-K + anti-C, anti-K + anti-c, anti-K + anti-Jka, anti-K + anti-C + anti-E, anti-K + anti-C + anti-Le ${ }^{a}$, anti-K + anti-C + anti-Jk ${ }^{\mathrm{a}}$, anti-K + anti-E + anti-Fy ${ }^{\mathrm{a}}$ e anti-E + anti-D + anti-Le ${ }^{\mathrm{a}}$

Do total de aloimunizados, 126 (72,83\%) eram do sexo feminino e $47(27,17 \%)$ do masculino $(2,7$ mulheres para 1 homem). A proporção do anti-D nas mulheres foi significativamente superior à dos homens $(\mathrm{p}=0,011)$. Porém, comparando as proporções dos demais anticorpos entre ambos os sexos, incluindo as associações, pode-se afirmar que não houve diferença estatisticamente significante (Tabela 2).

A classificação quanto à cor foi avaliada em 132 pacientes, dos quais 93 (70,45\%) eram brancos e 39 (29,54\%) nãobrancos. Comparando as proporções de anticorpos do sistema Rh (anti-D, anti-E, anti-c e anti-C) entre as duas raças, pode-se afirmar que não houve diferença estatisticamente significante, sendo que o mesmo é válido para os anticorpos dos demais sistemas e também para as associações de anticorpos (Tabela 3). 
Tabela 2. Principais anticorpos irregulares identificados em 173 pacientes, divididos por sexo, no HRU, de 1997 a 2005

\begin{tabular}{|c|c|c|c|c|c|}
\hline \multirow[t]{2}{*}{ Tipo de anticorpo } & \multicolumn{2}{|c|}{ masculino } & \multicolumn{2}{|c|}{ feminino } & \multirow[b]{2}{*}{$p$} \\
\hline & $\mathrm{n}^{\circ}$ & $\%$ & $\mathrm{n}^{\circ}$ & $\%$ & \\
\hline \multicolumn{6}{|l|}{ Sistema Rh } \\
\hline anti-D & 06 & 12,77 & 36 & 28,57 & 0,011 \\
\hline anti-E & 11 & 23,40 & 21 & 16,67 & 0,39 \\
\hline anti-c & 00 & 0,00 & 03 & 1,59 & 0,15 \\
\hline anti-C & 00 & 0,00 & 02 & 2,38 & 0,09 \\
\hline \multicolumn{6}{|l|}{ Outros sistemas } \\
\hline anti-K (Kell) & 11 & 23,40 & 13 & 10,32 & 0,06 \\
\hline anti-M (MNS) & 06 & 12,77 & 12 & 9,52 & 0,58 \\
\hline anti-Le $^{a}$ (Lewis) & 01 & 2,13 & 04 & 3,18 & 0,69 \\
\hline anti-Fya (Duffy) & 02 & 4,26 & 03 & 2,38 & 0,56 \\
\hline outros anticorpos* & 02 & 4,26 & 09 & 7,14 & 0,45 \\
\hline Associações ** & 08 & 17,02 & 28 & 22,22 & 0,45 \\
\hline Total & 47 & 100,00 & 126 & 100,00 & \\
\hline
\end{tabular}

*outros anticorpos: anti-Js ${ }^{a}$ e anti-N (masculino); anti-S, anti-Le ${ }^{b}$, anti-Jk ${ }^{\mathrm{a}}$ e anti-Jk ${ }^{\mathrm{b}}$ (feminino).

${ }^{* *}$ Associações: anti-D + anti-C, anti-e + anti-C, anti-E + anti-C, anti$\mathrm{E}+$ anti-K, anti-E + anti-Le ${ }^{\mathrm{a}}$, anti-C + anti-K, anti-E + anti-Jk ${ }^{\mathrm{a}}$ e anti-C + anti-K + anti-Le ${ }^{\text {a }}$ (masculino); anti-D + anti-C, anti-E + anti-C, anti$\mathrm{E}+$ anti-K, anti-E + anti-M, anti-E + anti-Fy ${ }^{\mathrm{a}}$, anti- $\mathrm{E}+$ anti-S, anti-K + anti-Le ${ }^{\mathrm{a}}$, anti-K + anti-C, anti-K + anti-Jk ${ }^{\mathrm{a}}$, anti-C + anti-Fy, anti-C + anti-Jk ${ }^{\mathrm{a}}$, anti-C + anti-K + anti-E, anti-C + anti-K + anti-Jk ${ }^{\mathrm{a}}$, anti-E + anti-Fy ${ }^{\mathrm{a}}+$ anti-K e anti-E + anti-D + anti-Le ${ }^{\mathrm{a}}$ (feminino)

Tabela 3. Principais anticorpos irregulares identificados em 132 pacientes, divididos por cor, no HRU, de 1997 a 2005

\begin{tabular}{|c|c|c|c|c|c|}
\hline \multirow[t]{2}{*}{ Tipo de anticorpo } & \multicolumn{2}{|c|}{ brancos } & \multicolumn{2}{|c|}{ não-brancos } & \multirow[b]{2}{*}{$p$} \\
\hline & $\mathrm{n}^{\circ}$ & $\%$ & $\mathrm{n}^{\circ}$ & $\%$ & \\
\hline \multicolumn{6}{|l|}{ Sistema Rh } \\
\hline anti-D & 23 & 24,73 & 09 & 23,08 & 0,81 \\
\hline anti-E & 17 & 18,28 & 09 & 23,08 & 0,60 \\
\hline anti-c & 02 & 0,02 & 01 & 0,03 & 0,74 \\
\hline anti-C & 02 & 0,02 & 00 & 0,00 & 0,15 \\
\hline \multicolumn{6}{|l|}{ Outros sistemas } \\
\hline anti-K (Kell) & 15 & 16,13 & 03 & 7,69 & 0,16 \\
\hline anti-M (MNS) & 08 & 8,60 & 05 & 12,82 & 0,50 \\
\hline anti-Le ${ }^{a}$ (Lewis) & 03 & 3,23 & 01 & 2,56 & 0,76 \\
\hline anti-Fya (Duffy) & 04 & 4,30 & 01 & 2,56 & 0,84 \\
\hline outros anticorpos* & 08 & 8,60 & 02 & 5,13 & 0,45 \\
\hline Associações** & 15 & 16,13 & 09 & 23,08 & 0,36 \\
\hline Total & 93 & 100,00 & 39 & 100,00 & \\
\hline
\end{tabular}

*outros anticorpos: anti-Js ${ }^{\mathrm{a}}$, anti-S, anti-Le ${ }^{\mathrm{b}}$, anti-Jk${ }^{\mathrm{a}}$ e anti-Jk ${ }^{\mathrm{b}}$ (brancos); anti-Jk (não-brancos).

**Associações: anti-D + anti-C, anti-E + anti-C, anti-E + anti-K, anti$\mathrm{E}+$ anti-Le ${ }^{\mathrm{a}}$, anti-C + anti-K, anti-E + anti-M, anti-K + anti-Le ${ }^{\mathrm{a}}$, anti-C + anti-E + anti-K, anti-C + anti-Le ${ }^{\mathrm{a}}+$ anti-K, anti-E + anti-Fy + anti-K e anti-E + anti-D + anti-Le (brancos); anti-C + anti-D, anti-E + anti$\mathrm{Jk}^{\mathrm{a}}$, anti-C + anti-Jk ${ }^{\mathrm{a}}$, anti-C + anti-e, anti-C + anti-E e anti-E + anti-s (não-brancos).
A distribuição segundo a faixa etária foi possível em 139 pacientes, dentre os quais $95(68,35 \%)$ tinham mais de 30 anos e $44(31,65 \%)$ estavam abaixo dessa idade, na data da identificação do anticorpo.

A classificação quanto ao tipo de doença em que foi necessário o tratamento hemoterápico foi avaliada em 73 pacientes, sendo que $29(39,73 \%)$ eram portadores de anemias de intercorrências agudas, 23 (31,51\%) de anemias de doenças crônicas e $21(28,77 \%)$ de doenças oncológicas ou oncohematológicas.

A distribuição segundo o número de transfusões, foi possível em 82 pacientes, dos quais 58 (70,73\%) tiveram seus anticorpos identificados até a $10^{\mathrm{a}}$ transfusão.

\section{Discussão}

No presente estudo, os anticorpos dos sistemas Rh e Kell foram os mais encontrados, o que está de acordo com a literatura. ${ }^{3-13,15} \mathrm{O}$ anticorpo mais freqüentemente identificado foi o anti-D $(24,28 \%)$, como em três outros estudos. ${ }^{2,5,9} \mathrm{Ob}$ servou-se ainda, concordando também com os achados da literatura, associações envolvendo mais de um tipo de anticorpo. ${ }^{2-15}$

Quanto ao sexo, as mulheres foram responsáveis por $72,83 \%$ dos pacientes em que foram identificados anticorpos irregulares, dados similares aos de vários outros estudos. ${ }^{4,9,14,15}$ Tais fatos podem ser explicados, entre outros fatores, pelas gestações, que constituem um importante risco de sensibilização. ${ }^{14}$ Todavia, contrariamente aos nossos achados, dois estudos não encontraram diferença significativa entre homens e mulheres. ${ }^{8,12}$

No sexo feminino, o anti-D foi o anticorpo mais freqüente, como também descrito por Baptista-González et al., ${ }^{2}$ que estudaram mulheres em idade reprodutiva. A incidência significativamente superior deste anticorpo no sexo feminino está de acordo com dois outros estudos. ${ }^{3,14}$ Tais achados podem ser também atribuídos às gestações, uma vez que o antígeno D é considerado o mais imunogênico nos casos de incompatibilidade materno-fetal. ${ }^{3}$

No presente estudo, não houve diferença significante na ocorrência de anticorpos do sistema $\mathrm{Rh}$ entre brancos e não-brancos, o que está de acordo com Coles et al. ${ }^{10}$

Analisando a faixa etária dos pacientes, a maioria $(68,35 \%)$ tinha mais de 30 anos de idade na data da identificação do anticorpo; dados similares aos de vários outros estudos. ${ }^{4,12,13,15}$ Tais resultados podem ser explicados por estarem esses indivíduos mais propensos a doenças, para os quais o tratamento hemoterápico se faz necessário. Como conseqüência, eles tornar-se-iam mais expostos à sensibilização eritrocitária, quando comparados aos mais jovens. Especificamente quanto às mulheres nesta faixa etária, a maioria já é multípara, o que contribui para aumentar o índice de sensibilização.

Dos 73 indivíduos em que a doença de base foi 
identificada, 39,73\% eram portadores de anemias de intercorências agudas, $31,51 \%$ de anemias de doenças crônicas e $28,77 \%$ de doenças oncológicas ou oncohematológicas. Schonewille et al. ${ }^{11}$ apesar de justificarem, com base em ampla revisão da literatura, que pacientes oncológicos, principalmente leucêmicos, possuem uma queda da resposta imunológica decorrente da própria doença de base e/ou da quimioterapia, encontraram, entre 1.066 pacientes com doenças linfo ou mieloproliferativas, uma freqüência de $9,0 \%$ de aloimunização eritrocitária. A implementação, na rotina do HRU, da fenotipagem prévia em todos os indivíduos passíveis de receberem múltiplas transfusões seguramente contribuiu para o menor índice de sensibilização dos pacientes oncológicos ou oncohematológicos, além dos portadores de doença falciforme e de anemia aplástica, com apenas quatro e um caso registrados, respectivamente. Chama ainda a atenção o fato de que os últimos cinco pacientes eram adultos e, portanto, já haviam sido transfundidos antes da implementação da fenotipagem na rotina do Serviço.

Por outro lado, a alta freqüência de portadores de anemias agudas entre os aloimunizados pode ser explicada pelo fato de que tais pacientes, pelas características de urgência em que as transfusões geralmente são indicadas, não se beneficiaram da fenotipagem eritrocitária pré-transfusional.

Com relação ao número de transfusões, dos 82 pacientes nos quais o número de unidades transfundidas pode ser adequadamente avaliado, $70,73 \%$ desenvolveram anticorpos antieritrocitários até a $10^{\mathrm{a}}$ transfusão, dado concordante com vários outros estudos. ${ }^{3,7-10,12}$ Segundo Blumberg et al., ${ }^{8}$ tais acontecimentos contrariam o conceito teórico de que a aloimunização eritrocitária é um fenômeno decorrente de transfusões crônicas, podendo-se então concluir que, na prática, ocorreria o oposto, ou seja, diversos pacientes tornar-se-iam aloimunizados durante suas exposições primárias aos antígenos. Tais achados sugerem que outros fatores devam contribuir para a sensibilização do paciente transfundido, como, por exemplo, a predisposição individual, possivelmente de caráter herdado, que poderia se manifestar já nas primeiras exposições ao antígeno estranho. Tal hipótese tem como substrato o fato de que cerca de $20 \%$ dos indivíduos não produzem anti-D, mesmo quando expostos várias vezes ao antígeno $\mathrm{D} \cdot{ }^{17}$

Em síntese, o índice relativamente baixo de pacientes sensibilizados no HRU $(0,75 \%)$, quando comparado aos dados da literatura, ${ }^{2,4,6-8,80-12,15}$ seguramente é conseqüência da implantação da fenotipagem eritrocitária no Serviço desde 1996, para os pacientes passíveis de receberem transfusões crônicas, como os portadores de doença falciforme, talassemias, anemia aplásica, doenças linfo e mieloproliferativas, renais crônicas e anemias refratárias. A implantação de tal rotina em todos os serviços de medicina transfusional contribuirá, sensivelmente, para aumentar a segurança transfusional em nosso país.

\begin{abstract}
Testing of the pre-transfusional blood phenotype, which has been carried out at the Regional Blood Bank in Uberaba since 1996, is an important procedure to improve safety of blood transfusions. This study aims to describe the frequency of irregular red blood cell antibodies in multiple-transfused patients from 1997 to 2005. In a retrospective study, data from all alloimmunized patients were collected from the blood bank files. Descriptive statistical analysis was performed and a comparison of proportions was made using the $Z$ test. Alloimmunization was observed in 173 (0.75\%) of the 23,220 transfused patients, with an average of 5.7 transfusions per patient. The frequency of the Rh and Kell systems jointly was over $70 \%$. The proportion of anti-D was significantly higher in women $(p<0.05)$ and no difference was noted in the Rh system between Caucasians and non-Caucasians. Seventy percent (70\%) of the patients were over 30 years of age. Out of the 73 patients with registered diseases, $39.73 \%$ had acute anemias, $31.51 \%$ chronic anemias and $28.77 \%$ oncological or onco-hematologic diseases. Approximately $70 \%$ of antibodies were discovered before the 10th transfusion. The low frequency of alloimmunization observed at the Regional Blood Bank of Uberaba reinforces the importance of pretransfusional blood phenotype screening for all multiple-transfused patients as well as its adoption as a common practice in all hemotherapy center. Rev. bras. hematol. hemoter. 2008;30(4):xxx$x x x$.
\end{abstract}

Key words: Alloimmunization; irregular antibodies; multitransfused patients.

\section{Agradecimentos}

A Ricardo Aparecido Olivo, professor auxiliar da disciplina de Hematologia e Hemoterapia da UFTM, e às biólogas Gisele Borges da Silva e Marieta Queluz, do HRU, pelo auxílio no levantamento dos dados publicados no estudo. À Maria Virgínia Goulart Borges de Lebron, professora de Lingua Inglesa da Central de Idiomas Modernos - UFTM è Gisele Marília Pianetti, bióloga da UFTM, pelas importantes sugestões na versão do resumo para o Inglês (abstract). À Fapemig (Fundação de Amparo à Pesquisa do Estado de Minas Gerais) e à Fundação Hemominas, pela concessão da Bolsa de Iniciação Científica.

\section{Referências Bibliográficas}

1. Jens E, Pagliarini T, Novaretti MCZ. Sistema de grupo sangüíneo Duffy: biologia e prática transfusional. Rev Bras Hematol Hemoter. 2005;27(2):110-19.

2. Baptista-González HA, Rosenfeld-Mann F, Pérez-Pérez JD, QuintanarGarcia E. Anticuerpos irregulares antieritrocitarios fuera del sistema ABO en el periodo perínatal. Bol Med Hosp Infant Mex 1991; 48(11):814-19.

3. García-Gala JM, Pabon MV, Vicente PR et al. Aloinmunización en pacientes politransfundidos. Utilidad de seleccionar hematíes compatibles para antígenos diferentes al ABO y D. Sangre 1994; 39(6):417-21.

4. Almeida Neto C, Mendonça MC, Braga MC et al. Aplicação do método gel LISS/COOMBS na rotina de pesquisa e identificação de 
anticorpos irregulares de pacientes. Serviços de Hemoterapia 9 de Julho, São Paulo - SP. Disponível em: http://www.hemoterapia9 dejulho.com.br/info_trab04.htm. Acesso em: 21/04/2006.

5. Ramos RJ, Cezarotto ES, Schorner EJ. Perfil dos anticorpos irregulares identificados no setor de Hematologia do Hemosc/Fpolis, de 08/2000 a 05/2003. Hemocentro de Santa Catarina/Hemosc. Disponível em: http://www.sbpc.org.br/site/pdf/100/ Hematologia.pdf. Acesso em: 10/05/2006.

6. Fluit CR, Kunst VA, Drenthe-Schonk AM. Incidence of red cell antibodies after multiple blood transfusion. Transfusion. 1990; 30(6):532-5.

7. Blumberg N, Peck K, Ross K, Avila E. Immune response to chronic red blood cell transfusion. Vox Sang. 1983;44(4):212-7.

8. Blumberg N, Ross K, Avila E, Peck K. Should chronic transfusions be matched for antigens other than $\mathrm{ABO}$ and Rho(D)? Vox Sang. 1984;47(3):205-8.

9. Walker RH, Lin DT, Hartrick MB. Alloimmunization following blood transfusion. Arch Pathol Lab Med. 1989;113(3):254-61.

10. Coles SM, Klein HG, Holland PV. Alloimmunization in two multitransfused patient populations. Transfusion. 1981;21(4):462-6.

11. Schonewille H, Haak HL, van Zijl AM. Alloimmunization after blood transfusion in patients with hematologic and oncologic diseases. Transfusion. 1999;39(7):763-71.

12. Redman M, Regan F, Contreras M. A Prospective study of the incidence of red cell allo-immunisation following transfusion. Vox Sang. 1996;71(4):216-20.

13. Schonewille H, Haak HL, van Zijl AM. RBC antibody persistence. Transfusion. 2000;40(9):1127-31.

14. Winters JL, Pineda AA, Gorden LD et al. RBC alloantibody specificity and antigen potency in Olmsted County, Minnesota. Transfusion. 2001;41(11):1413-20.

15. Schonewille H, van de Watering LM, Loomans DS, Brand A. Red blood cell alloantibodies after transfusion: factors influencing incidence and specificity. Transfusion. 2006;46(2):250-6.

16. Mattos LC. Duffy: um sistema de grupos sangüíneos com considerável complexidade. Rev Bras Hematol Hemoter. 2005;27(2):79-80.

17. Hematologia e Hemoterapia: fundamentos de morfologia, fisiologia, patologia e clínica. T Verrastro, TF Lorenzi, SW Neto. São Paulo: Editora Atheneu, 1998; 237-53.

18. A estatística básica e sua prática. DS Moore. Rio de Janeiro: LTC Editora, 2000.

Avaliação: Editor e dois revisores externos

Conflito de interesse: não declarado

Recebido: 10/03/2007

Aceito: $31 / 03 / 2008$ 